\title{
Randomized controlled trial of a clinical decision support system for painful polyneuropathy
}

\author{
Evan L. Reynolds $\mathrm{PhD}^{1}$ (0) ～James F. Burke MD, MS ${ }^{1,2} \quad$ | Mousumi Banerjee $\mathrm{PhD}^{3} \quad$ | \\ Brian C. Callaghan MD, MS ${ }^{1,2}$
}

${ }^{1}$ Health Services Research Program, Department of Neurology, University of Michigan, Ann Arbor, Michigan

${ }^{2}$ Veterans Affairs Healthcare System, Ann Arbor, Michigan

${ }^{3}$ School of Public Health, University of Michigan, Ann Arbor, Michigan

\section{Correspondence}

Brian Callaghan, 109 Zina Pitcher Place, 4021

BSRB, Ann Arbor, MI 48104.

Email: bcallagh@med.umich.edu

Funding information

National Institute of Neurological Disorders and Stroke, Grant/Award Numbers: K08 NS082597, R01 MD008879; National Institutes of Health, Grant/Award Number: K23 NS079417; U.S. Department of Veterans Affairs, Grant/Award Number: CSRD Merit CX001504

\begin{abstract}
Introduction: Despite the existence of guidelines, painful neuropathy is often inappropriately treated. We sought to determine the effectiveness of a clinical decision support system on guideline-recommended medication use.

Methods: We randomized neurology providers, stratified by subspecialty, to a best practice alert (BPA) linked to a Smartset or a BPA alone when seeing patients with neuropathy. The primary outcome was the proportion of patients with uncontrolled nerve pain prescribed a guideline-recommended medication. Generalized estimating equations were used to assess effectiveness.

Results: Seventy-five neurology providers (intervention 38, control 37) treated 2697 patients with neuropathy (intervention 1026, control 671). Providers did not acknowledge the BPA in 1928 (71.5\%) visits. Only four of eight intervention arm neurologists who treated patients with uncontrolled nerve pain opened the Smartset. The intervention was not associated with guideline-recommended medication use (odds ratio 0.52, 0.18-1.48; intervention 52\%, control 54.8\%).

Discussion: Our intervention did not improve prescribing practices for painful neuropathy. Physicians typically ignored the BPAs/Smartset; therefore, future studies should mandate their use or employ alternate strategies.

\section{KEYWORDS}

best practice alert, clinical decision support systems, neuropathic pain, neuropathy, opioid, randomized controlled trial
\end{abstract}

\section{1 | INTRODUCTION}

Neuropathy is a highly prevalent and painful condition. ${ }^{1-3}$ Recent reports indicate that tricyclic antidepressant drugs, serotonin norepinephrine update inhibitors, and gabapentinoids are efficacious for the treatment of neuropathic pain. ${ }^{4-7}$ Despite this robust evidence, we previously demonstrated that patients with neuropathy rarely receive more than one guideline-recommended medication. ${ }^{8}$ Furthermore, almost two-thirds of patients with neuropathy receive at least one opioid prescription, and nearly $9 \%$ receive chronic opioid therapy, often prior to any guideline-recommended medications. ${ }^{8}$ Because opioid treatment is associated with worse functional outcomes in patients with neuropathy, an intervention designed to increase guidelinerecommended medication use and decrease opioid use is essential. ${ }^{9}$

Clinical decision support systems (CDSS) could improve the use of guideline-recommended neuropathic pain medications while decreasing opioid use. Meta-analyses have demonstrated that CDSS interventions can improve physician behavior in diverse healthcare 
processes. ${ }^{10-13}$ We developed a CDSS that uses a best practice alert (BPA) linked to a Smartset (Epic, Verona, Wisconsin) to facilitate the ordering of guideline-recommended neuropathic pain medications and recommends against opioid treatment. We tested the effectiveness of the CDSS through a randomized, controlled trial (RCT).

\section{2 | MATERIALS AND METHODS}

\subsection{Simulation study and power calculation}

Prior to study implementation, we performed simulations to determine the appropriate sample size. We used 3 months of preliminary data from neurologists at the University of Michigan to estimate the frequency of patients with uncontrolled neuropathic pain, proportion of patients treated with guideline-recommended medications, and the typical number of patients treated per provider. We estimated $80.1 \%$ power to detect a $5 \%$ increase in guideline-recommended prescriptions for 1000 patients over 1 year using a generalized estimating equations (GEE) model with exchangeable correlation structure.

\subsection{Intervention}

Neurologists at the University of Michigan were provided study information through a presentation at a mandatory faculty meeting and several subsequent emails. Each provider was given an opportunity not to participate, but all chose to participate. The 103 neurologists were assigned to receive the BPA with or without the Smartset using block randomization, stratified by provider subspecialty (general neurologists $n=7$, neuromuscular specialists $n=4$, neurologists with specialties other than neuromuscular $n=46$, neurology fellows $n=19$, neurology residents $n=17$, and neurology nurse practitioners $n=10)$. Patients with neuropathy were identified by using ICD-10 codes (G60-G65, E08-11.40/42, E13.40/42, M79.2, A36.83, $\mathrm{B} 27.01 / 11 / 81 / 91, \mathrm{~B} 26.84, \mathrm{~B} 02.23, \mathrm{M} 34.83$ ) or when "peripheral neuropathy" was included as the chief complaint or in the problem summary list. When a patient with neuropathy met inclusion criteria, the BPA was automatically triggered with (intervention group) or without (control group) the Smartset. Figure S1A,B displays images of the BPA and Smartset, respectively. Providers received the BPA and then determined nerve pain status and entered medication status as follows (Figure S1A):

1. No nerve pain

2. Well controlled nerve pain, off medication

3. Well controlled nerve pain, on medication

4. Uncontrolled nerve pain

When the patient had uncontrolled nerve pain, the intervention group would receive a link to the Smartset, which gave information involving guideline-recommended medications including dosage information, typical medication pricing, advice to avoid opioid medication use, and a link to the
American Academy of Neurology guidelines (Figure S1B). ${ }^{5}$ Both the BPA and the Smartset were delivered through the electronic medical record used at the University of Michigan (Epic, Verona, Wisconsin).

\section{3 | Outcomes}

The primary outcome was the proportion of patients with uncontrolled nerve pain that were prescribed a guideline-recommended medication. The secondary outcome was the proportion of patients with uncontrolled nerve pain that were prescribed an opioid. To understand the use of our CDSS, we collected two process outcomes, the proportion of BPAs acknowledged and the proportion of Smartsets opened.

\subsection{Statistical analysis}

Descriptive statistics were used to characterize patients with uncontrolled nerve pain. We report the frequencies that guidelinerecommended medications, opioids, or other potential neuropathic pain medications were prescribed. The primary analysis used GEE with a logit link to assess the effects of the intervention on guidelinerecommended prescriptions. In addition to adjusting for patient factors (age, sex, race, insurance plan type) and provider subspecialty, the GEE approach accounts for clustering at the neurologist level because the same provider may treat multiple patients. Data analysis was completed in Rv.3.4.2 (R Foundation for Statistical Computing, Austria, Vienna). This study was approved by the University of Michigan Institutional Review Board (HUM00109137).

\section{$3 \mid$ RESULTS}

Between July 14, 2016 and July 13, 2017, 75 neurology providers (intervention 38 [50.7\%], control 37 [49.3\%]) treated 2697 patients with neuropathy (intervention 1026 [38\%], control 1671 [62\%]). Providers did not acknowledge the BPA in 1928 (71.5\%) visits (intervention 789 [77.3\%], control 1139 [68\%]). When the BPA was acknowledged, $6.9 \%$ of patients had controlled nerve pain without medication (intervention 14 [5.4\%], control 39 [6.7\%]), $27.2 \%$ of patients had controlled nerve pain with medication (intervention 7 [30.2\%], control 131 [22.4\%]), 37.6\% of patients had no nerve pain (intervention 99 [38.5\%], control 190 [32.5\%]), and $28.4 \%$ of patients had uncontrolled nerve pain (intervention 41 [15.9\%], control 177 [30.3\%]). There were eight neurologists in the intervention arm and 20 in the control arm that treated patients with uncontrolled nerve pain. Only four of eight neurologists in the intervention arm (25/41 patients) opened the Smartset during follow-up.

Demographic, health plan, and provider subspecialty for patients with uncontrolled nerve pain is presented in Table 1. Despite stratifying by provider subspecialty, we observed different patterns among 
TABLE 1 Patients' demographic, health plan, and provider information

\begin{tabular}{|c|c|c|}
\hline Variables & $\begin{array}{l}\text { Intervention } \\
\text { patients, } n=25\end{array}$ & $\begin{array}{l}\text { Control } \\
\text { patients, } n=177\end{array}$ \\
\hline Age, mean $\pm S D, y$ & $58.3 \pm 15.2$ & $56.6 \pm 13.9$ \\
\hline Men, n (\%) & $17(68)$ & $73(41.2)$ \\
\hline \multicolumn{3}{|l|}{ Race } \\
\hline White & $23(92)$ & $158(89.3)$ \\
\hline Black & $2(8)$ & $15(8.5)$ \\
\hline Asian & $0(0)$ & $2(1.1)$ \\
\hline Other & $0(0)$ & $2(1.1)$ \\
\hline $\begin{array}{l}\text { Ethnicity Hispanic, n } \\
\text { (\%) }\end{array}$ & $0(0)$ & $6(3.4)$ \\
\hline \multicolumn{3}{|l|}{ Health plan, n (\%) } \\
\hline Medicare & $9(36)$ & 55 (31.1) \\
\hline $\begin{array}{l}\text { Blue Cross Blue } \\
\text { Shield }\end{array}$ & $8(32)$ & $54(30.5)$ \\
\hline Blue Care Network & $2(8)$ & $20(11.3)$ \\
\hline Priority Health & $1(4)$ & $13(7.3)$ \\
\hline $\begin{array}{l}\text { Meridian Health } \\
\text { Plan }\end{array}$ & $3(12)$ & $8(4.5)$ \\
\hline Mclaren & $0(0)$ & $6(3.4)$ \\
\hline United Healthcare & $0(0)$ & $5(2.8)$ \\
\hline Other & $2(8)$ & $16(9)$ \\
\hline \multicolumn{3}{|l|}{$\begin{array}{l}\text { Provider subspecialty, } \\
\mathrm{n}(\%)\end{array}$} \\
\hline General neurology & $0(0)$ & $61(34.5)$ \\
\hline Resident & $22(88)$ & 37 (20.9) \\
\hline Neuromuscular & $0(0)$ & $39(22)$ \\
\hline Fellows & $0(0)$ & $31(17.5)$ \\
\hline $\begin{array}{l}\text { Attending } \\
\text { neurologist }\end{array}$ & $3(12)$ & $9(5.1)$ \\
\hline Nurse practioner & $0(0)$ & $0(0)$ \\
\hline
\end{tabular}

providers who treated patients with uncontrolled nerve pain. Patients in the intervention arm were treated by residents and attending neurologists. Patients in the control arm were treated by general neurologists, neuromuscular specialists, residents, fellows, and attending neurologists.

Frequencies of relevant medications that were prescribed to patients are presented in Table 2 . The proportion of patients receiving guideline-recommended medications was similar in the intervention and control arms. No patients were prescribed an opioid in the intervention arm compared with 11 patients in the control arm.

The GEE revealed that the intervention was not associated with guideline-recommended medication use (crude odds ratio [OR] 0.89 , 0.36-2.24; adjusted OR 0.52, 0.18-1.48). Men (adjusted OR 2.10, 1.14-3.89) and patients treated by residents (adjusted OR 2.18, 1.12-5.66, reference $=$ general neurologists) had an increased odds of guideline-recommended medication use. Insurance type, patient race, and age were not significantly associated with guideline-
TABLE 2 Frequency of neuropathic pain medication prescriptions

\begin{tabular}{|c|c|c|}
\hline Medications & $\begin{array}{l}\text { Intervention, } \\
\mathrm{n}(\%)\end{array}$ & $\begin{array}{l}\text { Control, } \\
\text { n (\%) }\end{array}$ \\
\hline $\begin{array}{l}\text { Guideline-recommended } \\
\text { medications }\end{array}$ & $13(52)$ & $97(54.8)$ \\
\hline Gabapentin & $4(16)$ & $40(22.6)$ \\
\hline Nortriptyline & $5(20)$ & $27(15.3)$ \\
\hline Pregabalin & $2(8)$ & $15(8.5)$ \\
\hline Duloxetine & $1(4)$ & $15(8.5)$ \\
\hline Amitriptyline & $0(0)$ & $10(5.7)$ \\
\hline Venlafaxine & $1(4)$ & $3(1.7)$ \\
\hline Doxepin & $0(0)$ & $2(1.1)$ \\
\hline Opioids & $0(0)$ & $11(6.2)$ \\
\hline Oxycodone & $0(0)$ & $6(3.4)$ \\
\hline Methadone & $0(0)$ & $3(1.7)$ \\
\hline Morphine & $0(0)$ & $3(1.7)$ \\
\hline Buprenorphine, naloxone & $0(0)$ & $2(1.1)$ \\
\hline Fentanyl & $0(0)$ & $1(0.6)$ \\
\hline Hydrocodone & $0(0)$ & $1(0.6)$ \\
\hline \multicolumn{3}{|c|}{ Other potential pain medications } \\
\hline Tramadol & $0(0)$ & $14(7.9)$ \\
\hline Topiramate & $1(4)$ & $7(4)$ \\
\hline Zonisamide & $0(0)$ & $8(4.5)$ \\
\hline Carbamazepine & $0(0)$ & $7(4)$ \\
\hline Lamotrigine & $0(0)$ & $7(4)$ \\
\hline Baclofen & $2(8)$ & $4(2.3)$ \\
\hline Levetiracetam & $0(0)$ & $5(2.8)$ \\
\hline Lidocaine & $1(0)$ & $4(2.3)$ \\
\hline Other & $4(16)$ & $13(7.3)$ \\
\hline
\end{tabular}

recommended medication use. We were unable to fit a GEE model for the secondary outcome because there were no opioids prescribed in the intervention arm.

\section{4 | DISCUSSION}

Our intervention failed to improve either the primary or secondary outcome measures. Future interventions should be informed by the lessons learned from our negative trial. Our CDSS failed in two major capacities. First, our process outcomes indicated that physicians usually did not acknowledge the BPA or use the Smartset intervention. The low use rate resulted in an insufficient sample size to assess the effectiveness of the intervention. Our observed low use is not unusual; a previous meta-analysis found that most RCTs (8/12) observed poor physician use of CDSS interventions (however, this information was rarely reported). ${ }^{11}$ One solution involves implementing a mandatory BPA with an automatically fired Smartset. A mandatory-response BPA would improve CDSS use; however, previous researchers found no difference in the rate by which physicians accepted the CDSS 
recommendation when responses were required. ${ }^{11}$ Therefore, it is unclear whether higher use would improve prescribing patterns. Rather than a mandatory response CDSS, other strategies could be employed to incentivize providers to use the intervention through financial bonuses or other means. ${ }^{14}$ Furthermore, the intervention may have led to alert fatigue. ${ }^{15}$ One potential solution is to focus future interventions on patients that self-report pain and/or are not taking current guideline-recommended medications. Finally, embedding a predictive tool into the BPA to help determine which patients would most benefit from a specific medication could increase the perceived utility of the CDSS. $^{16,17}$ Future CDSS interventions should (1) implement a more intensive implementation strategy to increase provider participation or (2) plan for low use rates when determining sample size and follow-up length. The second study shortcoming was that the distribution of patients in the two arms of our trial was asymmetric despite stratification by provider type. To mitigate this issue, future studies could increase the number of physicians randomized through a multicenter study or stratify physicians on the basis of previous frequencies of outpatient neuropathy visits.

Changing physician behavior is difficult, even when implementing a CDSS that follows the typical workflow for ordering medications. One possible solution would be to target physicians with less experience, such as residents. Unfortunately, previous meta-analyses have found no association between physician experience and CDSS intervention effectiveness. ${ }^{12,18,19}$ Our finding that residents have higher rates of CDSS use warrants further study.

Study limitations include the small sample and the asymmetric distribution of physicians in each group. Given the small sample, we were unable to account for the nested, networked nature of trainees being supervised by different attending neurologists. Whether our results are generalizable to other provider specialties is unclear. We did not have baseline data from the time period immediately prior to the intervention; therefore, we do not know whether the two groups were balanced at baseline in terms of medication use. This study was unable to address whether the intervention would be successful with mandatory BPAs.

Our proposed CDSS was unsuccessful, both in its use and in altering prescribing patterns of guideline-recommended medications. Performing RCTs to assess the effectiveness of CDSS interventions is essential. Lack of rigorous testing may lead to ineffective CDSS that add unnecessary work to physicians. Our negative trial allowed us to delete this BPA and lessen the burden on neurologists at the University of Michigan.

\section{CONFLICT OF INTEREST}

Brian Callaghan receives research support from Impeto Medical Inc. He performs medical consultations for Advance Medical, consults for a PCORI grant, consults for the immune tolerance network, and performs medical legal consultations. James Burke has received compensation from Astra Zeneca for his role on the adjudication committee of the SOCRATES trial. Evan Reynolds and Mousumi Banerjee report no conflicts of interest related to this report.

\section{ETHICAL PUBLICATION STATEMENT}

We confirm that we have read the Journal's position on issues involved in ethical publication and affirm that this report is consistent with those guidelines.

\section{ORCID}

Evan L. Reynolds (D) https://orcid.org/0000-0002-0138-8436

\section{REFERENCES}

1. Daousi C, MacFarlane I, Woodward A, Nurmikko T, Bundred P, Benbow S. Chronic painful peripheral neuropathy in an urban community: a controlled comparison of people with and without diabetes. Diabet Med. 2004;21(9):976-982.

2. Gregg EW, Sorlie P, Paulose-Ram R, et al. Prevalence of lowerextremity disease in the US adult population $\geq 40$ years of age with and without diabetes: 1999-2000 national health and nutrition examination survey. Diabetes Care. 2004;27(7):1591-1597.

3. Savettieri G, Rocca WA, Salemi G, et al. Prevalence of diabetic neuropathy with somatic symptoms: a door-to-door survey in two Sicilian municipalities. Sicilian Neuro-Epidemiologic Study (SNES) Group. Neurology. 1993;43(6):1115-1120.

4. Attal N, Cruccu G, Baron R al, et al. EFNS guidelines on the pharmacological treatment of neuropathic pain: 2010 revision. Eur J Neurol. 2010;17(9):1113-e88.

5. Bril V, England J, Franklin GM, et al. Evidence-based guideline: treatment of painful diabetic neuropathy: report of the American Academy of Neurology, the American Association of Neuromuscular and Electrodiagnostic Medicine, and the American Academy of Physical Medicine and Rehabilitation. P MR. 2011;3(4):345-352.

6. Finnerup NB, Attal N, Haroutounian S, et al. Pharmacotherapy for neuropathic pain in adults: a systematic review and meta-analysis. Lancet Neurol. 2015;14(2):162-173.

7. Griebeler ML, Morey-Vargas OL, Brito JP, et al. Pharmacologic interventions for painful diabetic neuropathy: an umbrella systematic review and comparative effectiveness network meta-analysis. Ann Intern Med. 2014;161(9):639-649.

8. Callaghan BC, Reynolds E, Banerjee M, Kerber KA, Skolarus LE, Burke JF. Longitudinal pattern of pain medication utilization in peripheral neuropathy patients. Pain. 2019;160(3):592-599.

9. Hoffman EM, Watson JC, St Sauver J, Staff NP, Klein CJ. Association of long-term opioid therapy with functional status, adverse outcomes, and mortality among patients with polyneuropathy. JAMA Neurol. 2017;74(7):773-779. https://doi.org/10.1001/jamaneurol.2017.0486.

10. Holstiege J, Mathes T, Pieper D. Effects of computer-aided clinical decision support systems in improving antibiotic prescribing by primary care providers: a systematic review. J Am Med Inform Assoc. 2014;22(1):236-242.

11. Bright TJ, Wong A, Dhurjati R, et al. Effect of clinical decision-support systems: a systematic review. Ann Intern Med. 2012;157(1):29-43.

12. Garg AX, Adhikari NK, McDonald H, et al. Effects of computerized clinical decision support systems on practitioner performance and patient outcomes: a systematic review. JAMA. 2005;293(10):1223-1238.

13. Pearson S-A, Moxey A, Robertson J, et al. Do computerised clinical decision support systems for prescribing change practice? A systematic review of the literature (1990-2007). BMC Health Serv Res. 2009; 9(1):154.

14. Ballard DW, Vemula R, Chettipally UK, et al. Optimizing clinical decision support in the electronic health record. clinical characteristics associated with the use of a decision tool for disposition of ED patients with pulmonary embolism. Appl Clin Inform. 2016;7(3):883-898. https://doi.org/10.4338/ACI-2016-05-RA0073. 
15. Coleman JJ, van der Sijs $\mathrm{H}$, Haefeli WE, et al. On the alert: future priorities for alerts in clinical decision support for computerized physician order entry identified from a European workshop. BMC Med Inform Decis Mak. 2013;13:111. https://doi.org/10.1186/1472-694713-111.

16. McGinn TG, McCullagh L, Kannry J, et al. Efficacy of an evidence-based clinical decision support in primary care practices: a randomized clinical trial. JAMA Intern Med. 2013;173(17):15841591.

17. Gonzales R, Anderer T, McCulloch CE, et al. A cluster randomized trial of decision support strategies for reducing antibiotic use in acute bronchitis. JAMA Intern Med. 2013;173(4):267-273. https://doi.org/ 10.1001/jamainternmed.2013.1589.

18. Vissers $M C$, Hasman A, van der Linden CJ. Impact of a protocol processing system (ProtoVIEW) on clinical behaviour of residents and treatment. Int J Biomed Comput. 1996;42(1-2):143-150.
19. Demakis JG, Beauchamp C, Cull WL, et al. Improving residents' compliance with standards of ambulatory care: results from the VA Cooperative Study on Computerized Reminders. JAMA. 2000;284(11):1411-1416.

\section{SUPPORTING INFORMATION}

Additional supporting information may be found online in the Supporting Information section at the end of this article.

How to cite this article: Reynolds EL, Burke JF, Banerjee M, Callaghan BC. Randomized controlled trial of a clinical decision support system for painful polyneuropathy. Muscle \& Nerve. 2020;61:640-644. https://doi.org/10.1002/mus.26774

\title{
Magnetic resonance imaging correlates with electrical impedance myography in facioscapulohumeral muscular dystrophy
}

\author{
Johanna Hamel MD $\quad$ ～Phil Lee PhD ${ }^{2,3} \quad$ | Melanie D. Glenn MD ${ }^{4}$ | \\ Tekalign Burka MD ${ }^{4}$ | In-Young Choi PhD ${ }^{3,4}$ | Seth D. Friedman $\mathrm{PhD}^{5}$ | \\ Dennis W. W. Shaw MD ${ }^{5}$ | Ayla McCalley BA ${ }^{4}$ | Laura Herbelin CCRP ${ }^{4}$ | \\ Mazen M. Dimachkie MD ${ }^{4}$ | Richard Lemmers PhD ${ }^{6}$ | \\ Silvère M. van der Maarel $\mathrm{PhD}^{6} \quad$ ～Richard J. Barohn $\mathrm{MD}^{4} \quad$ | Rabi Tawil MD ${ }^{1}$ | \\ Jeffrey M. Statland MD ${ }^{4}$ (i)
}

\footnotetext{
${ }^{1}$ Department of Neurology, University of Rochester Medical Center, Rochester, New York

${ }^{2}$ Department of Radiology, University of Kansas Medical Center, Kansas City, Kansas

${ }^{3}$ Hoglund Brain Imaging Center, University of Kansas Medical Center, Kansas City, Kansas

${ }^{4}$ Department of Neurology, University of Kansas Medical Center, Kansas City, Kansas

${ }^{5}$ Center for Clinical and Translational Research, Seattle Children's Hospital, Seattle, Washington

${ }^{6}$ Department of Human Genetics, Leiden University Medical Center, Leiden, The Netherlands
}

\begin{abstract}
Introduction: Electrical impedance myography (EIM) has been proposed as a noninvasive biomarker of muscle composition in facioscapulohumeral muscular dystrophy (FSHD). Here we determine the associations of EIM variables with muscle structure measured by MRI.

Methods: We evaluated 20 patients with FSHD at two centers, comparing EIM measurements (resistance, reactance, and phase at 50,100, and $211 \mathrm{kHZ}$ ) recorded from bilateral vastus lateralis, tibialis anterior, and medial gastrocnemius muscles to MRI skin and subcutaneous fat thickness, MRI T1-based muscle severity score (T1 muscle score), and MRI quantitative intramuscular Dixon fat fraction (FF).
\end{abstract}

